MATHEMATICS OF COMPUTATION

Volume 74, Number 252, Pages 1777-1791

S 0025-5718(05)01748-5

Article electronically published on February 16, 2005

\title{
ALGORITHMS \\ FOR HYPERBOLIC QUADRATIC EIGENVALUE PROBLEMS
}

\author{
CHUN-HUA GUO AND PETER LANCASTER
}

\begin{abstract}
We consider the quadratic eigenvalue problem (or the QEP) $\left(\lambda^{2} A+\lambda B+C\right) x=0$, where $A, B$, and $C$ are Hermitian with $A$ positive definite. The QEP is called hyperbolic if $\left(x^{*} B x\right)^{2}>4\left(x^{*} A x\right)\left(x^{*} C x\right)$ for all nonzero $x \in \mathbb{C}^{n}$. We show that a relatively efficient test for hyperbolicity can be obtained by computing the eigenvalues of the QEP. A hyperbolic QEP is overdamped if $B$ is positive definite and $C$ is positive semidefinite. We show that a hyperbolic QEP (whose eigenvalues are necessarily real) is overdamped if and only if its largest eigenvalue is nonpositive. For overdamped QEPs, we show that all eigenpairs can be found efficiently by finding two solutions of the corresponding quadratic matrix equation using a method based on cyclic reduction. We also present a new measure for the degree of hyperbolicity of a hyperbolic QEP.
\end{abstract}

\section{INTRODUCTION}

Quadratic eigenvalue problems (QEPs) appear in many applications. For a recent survey on this topic, see 21]. The QEP is to find scalars $\lambda$ and nonzero vectors $x$ satisfying

$$
Q(\lambda) x=\left(\lambda^{2} A+\lambda B+C\right) x=0, \quad A, B, C \in \mathbb{C}^{n \times n} .
$$

The vectors $x$ are the eigenvectors corresponding to the eigenvalues $\lambda$. We will limit our attention to QEPs where $A, B, C$ are Hermitian and $A$ is positive definite. We denote by $\mathcal{H}_{n}$ the set of all $n \times n$ Hermitian matrices. For $W_{1}, W_{2} \in \mathcal{H}_{n}$, we write $W_{1} \geq W_{2}\left(W_{1}>W_{2}\right)$ if $W_{1}-W_{2}$ is positive semidefinite (definite). Hyperbolic and elliptic QEPs are disjoint classes of QEPs (see [16]).

In a recent paper by Higham, Tisseur, and Van Dooren [13, methods for detecting a hyperbolic or elliptic QEP are discussed. They point out that testing for ellipticity can be done straightforwardly by computing the eigenvectors of the QEP, while testing for hyperbolicity is more complicated. They then show that testing for hyperbolicity can be reduced to testing for definiteness of a Hermitian pencil of twice the dimension. In Section 2 of this paper, we show that testing for hyperbolicity can be done by computing all $2 n$ eigenvalues of the QEP. In particular, testing for hyperbolicity is actually half as expensive as testing for ellipticity. Our

Received by the editor August 7, 2003 and, in revised form, June 6, 2004.

2000 Mathematics Subject Classification. Primary 65F30; Secondary 15A18, 15 A24.

Key words and phrases. Quadratic eigenvalue problem, overdamping condition, quadratic matrix equation, solvent, cyclic reduction.

This work was supported in part by grants from the Natural Sciences and Engineering Research Council of Canada. 
method is less expensive than the method proposed in [13] since that method needs to compute all $4 n$ eigenvalues of a $2 n \times 2 n$ QEP with complex matrices (even if the matrices $A, B$ and $C$ are all real). Since hyperbolic QEPs include the class of overdamped QEPs, our method also provides a relatively easy way to check Duffin's overdamping condition [6].

We also mention that the hyperbolicity of a QEP is related to the stability of the gyroscopic system $L(\lambda)=\lambda^{2} A_{g}+\lambda B_{g}+C_{g}$, where $A_{g}, C_{g}>0$ and $B_{g}$ is Hermitian indefinite and invertible. It is well known [2] that the system is stable whenever $\left|B_{g}\right|>k A_{g}+k^{-1} C_{g}$ for some real $k>0$, where $\left|B_{g}\right|$ is the positive definite square root of $\left(B_{g}\right)^{2}$. In other words, the gyroscopic system is stable whenever the QEP $\left(\lambda^{2} A_{g}+\lambda\left|B_{g}\right|+C_{g}\right) x=0$ is hyperbolic (Theorem 2 of Section 2 is used here). Thus, a method for testing hyperbolicity is also a method for verifying the sufficient condition for the stability of gyroscopic systems. We note that the sufficient condition can be replaced by a weaker sufficient condition 17. However, the latter condition is more difficult to verify, and it is still related to the hyperbolicity condition in some sense (see Theorem 2 of [17]).

The QEP (1) is closely related to the matrix equation

$$
Q(X)=A X^{2}+B X+C=0
$$

whose solutions are also called the solvents of $Q(X)$. For an overdamped QEP, the eigenvalues are real, semisimple, and nonpositive; there is a gap between the $n$ largest eigenvalues (the primary eigenvalues) and the $n$ smallest eigenvalues (the secondary eigenvalues); and $Q(X)$ has at least two solvents $X^{(1)}$ and $X^{(2)}$, having as their eigenvalues the primary eigenvalues and the secondary eigenvalues, respectively. See [6] and [15] for more details. We will refer to the solvents $X^{(1)}$ and $X^{(2)}$ as the primary and the secondary solvents.

As noted in [13, a hyperbolic QEP becomes an overdamped QEP after a proper shift is applied to the eigenvalue parameter. It is then easy to see that the aforementioned properties for an overdamped QEP also hold for a hyperbolic QEP except that the eigenvalues are not necessarily nonpositive. In Section 2, we point out that a hyperbolic QEP is overdamped if and only if all its eigenvalues are nonpositive.

The standard approach for finding the eigenvalues/eigenvectors of the QEP (11) is by linearizations. However, for overdamped systems, solving the QEP (11) by computing the primary and the secondary solvents can be more efficient. Indeed, Higham and Kim [11 have already shown that the solvent approach can be more efficient than the linearization approach when the two solvents are found by a Bernoulli iteration, provided that the gap between the primary and the secondary eigenvalues is large. We show in Section 3 that the solvent approach is usually more efficient than the linearization approach when the two solvents are computed by the cyclic reduction method, even if the gap between the primary and the secondary eigenvalues is small. The advantage of the solvent approach is more conspicuous when the more expensive QZ algorithm (rather than the QR algorithm) is needed for the linearization approach, and when both eigenvalues and eigenvectors are required. The solvent approach also leads to an even faster method for checking the overdamping condition in Section 4. In Section 5, we show that the hyperbolicity is lost exactly when the gap between the primary and the secondary eigenvalues is lost. The gap is thus an easily computable measure for the degree of hyperbolicity of a hyperbolic QEP. 


\section{TeSting FOR HYPerbolicity By COMPUting $2 n$ EIGENVAlues}

The class of hyperbolic QEPs is defined as follows [16].

Definition 1. The $Q E P$ (11) is hyperbolic if $A, B, C \in \mathcal{H}_{n}, A>0$, and

$$
\left(x^{*} B x\right)^{2}>4\left(x^{*} A x\right)\left(x^{*} C x\right) \text { for all nonzero } x \in \mathbb{C}^{n} \text {. }
$$

The next result follows from Lemmas 31.15 and 31.23 in [18]. For an alternative proof, see [1].

Theorem 2. $A$ QEP with $A, B, C \in \mathcal{H}_{n}$ and $A>0$ is hyperbolic if and only if $Q(\mu)<0$ for some $\mu \in \mathbb{R}$.

According to this result, testing for hyperbolicity can be done by solving the one-dimensional global optimization problem $\min _{\mu} \lambda_{\max }(Q(\mu))$. It has been noted in [13] that testing for hyperbolicity can be reduced to testing for definiteness of a Hermitian pencil of twice the dimension. Here we point out that testing for hyperbolicity can be done more easily. In fact:

Theorem 3. The $Q E P$ (11) with $A, B, C \in \mathcal{H}_{n}$ and $A>0$ is hyperbolic if and only if

$$
Q(\lambda) \text { has } 2 n \text { real eigenvalues } \lambda_{1} \geq \cdots \geq \lambda_{n}>\lambda_{n+1} \geq \cdots \geq \lambda_{2 n}
$$

and

$$
Q(\lambda)<0 \text { for some (equivalently all) } \lambda \in\left(\lambda_{n+1}, \lambda_{n}\right) .
$$

Proof. The "if" part is true by Theorem 2, For the "only if" part, (3) is already known. We now prove (4). For each $\lambda \in \mathbb{R}, Q(\lambda)$ is a Hermitian matrix and thus has $n$ real eigenvalues $\mu_{1}(\lambda), \ldots, \mu_{n}(\lambda)$. These $n$ eigenvalues are continuously dependent on $\lambda$. We know that $\lambda_{k}$ is an eigenvalue of $Q(\lambda)$ if and only if 0 is an eigenvalue of the matrix $Q\left(\lambda_{k}\right)$. Since $Q\left(\lambda_{k}\right)$ is Hermitian, the algebraic multiplicity of 0 as an eigenvalue of $Q\left(\lambda_{k}\right)$ is equal to its geometric multiplicity, which is in turn equal to the geometric multiplicity of $\lambda_{k}$ as an eigenvalue of $Q(\lambda)$. (This can be seen easily by using the local Smith form of $Q(\lambda)$ at $\lambda_{k}$; see [7, for example.) Since $\lambda_{k}$ is a semisimple eigenvalue of $Q(\lambda)$, it follows that the algebraic multiplicity of 0 as an eigenvalue of $Q\left(\lambda_{k}\right)$ is equal to the algebraic multiplicity of $\lambda_{k}$ as an eigenvalue of $Q(\lambda)$. Since $A>0, Q(\lambda)>0$ for any $\lambda$ with sufficiently large absolute value. Therefore, $Q(\lambda)>0$ for $\lambda \in\left(-\infty, \lambda_{2 n}\right)$ and for $\lambda \in\left(\lambda_{1}, \infty\right)$. Thus, $Q(\lambda)$ has $n$ positive eigenvalues for each $\lambda>\lambda_{1}$. We now decrease $\lambda$. If $\lambda_{1}=\cdots=\lambda_{p}>$ $\lambda_{p+1}(1 \leq p \leq n)$, then 0 is an eigenvalue of $Q\left(\lambda_{1}\right)$ with algebraic multiplicity $p$. Therefore, when $\lambda$ crosses the point $\lambda_{1}$, at most $p$ eigenvalues of $Q(\lambda)$ change from positive to negative. We then move $\lambda$ further toward $\lambda_{n+1}$ and conclude that the number of negative eigenvalues of $Q(\lambda)$ is less than $n$ for $\lambda \in\left[\lambda_{n}, \infty\right)$. Similarly, we can increase $\lambda$ from the left of $\lambda_{2 n}$ toward $\lambda_{n}$ and conclude that the number of negative eigenvalues of $Q(\lambda)$ is also less than $n$ for $\lambda \in\left(-\infty, \lambda_{n+1}\right]$. Since $Q(\lambda)<0$ for some $\lambda \in \mathbb{R}$, we conclude that $Q(\lambda)$ has $n$ negative eigenvalues for some (and actually all) $\lambda \in\left(\lambda_{n+1}, \lambda_{n}\right)$.

The proof of the above theorem is quite standard. Similar arguments have been used in [7] and [14. From the proof we can see that $Q(\lambda)<0$ holds for all $\lambda \in\left(\lambda_{n+1}, \lambda_{n}\right)$ and for no other values of $\lambda$. Therefore, if a QEP is actually 
hyperbolic with $\lambda_{n} \approx \lambda_{n+1}$, it would be very difficult to confirm the hyperbolicity by trial and error to see if $Q(\lambda)<0$ for some $\lambda \in \mathbb{R}$.

The above theorem gives a numerical procedure to determine the hyperbolicity of the QEP. It is more efficient than the one proposed in [13].

Algorithm 1. Compute the $2 n$ eigenvalues of the QEP (1).

If they do not satisfy (3), then the QEP is not hyperbolic.

If they satisfy (3) and $Q\left(\left(\lambda_{n}+\lambda_{n+1}\right) / 2\right)<0$, then the QEP is hyperbolic; otherwise it is not hyperbolic.

Methods for finding the $2 n$ eigenvalues of the QEP will be discussed in Section 3. To see if $W=Q\left(\left(\lambda_{n}+\lambda_{n+1}\right) / 2\right)<0$, we can determine whether $-W$ has a Cholesky factorization or whether the largest eigenvalue of $W$ is negative.

Overdamped QEPs are special hyperbolic QEPs.

Definition 4. The $Q E P$ (11) is overdamped if it is hyperbolic and $B>0, C \geq 0$.

As noted in [13], a hyperbolic problem becomes an overdamped problem if a proper shift is used. Indeed, for any real number $\theta$,

$$
\begin{aligned}
Q(\lambda+\theta) & =\lambda^{2} A+\lambda(B+2 \theta A)+C+\theta B+\theta^{2} A \\
& =\lambda^{2} \tilde{A}+\lambda \tilde{B}+\tilde{C}=\tilde{Q}(\lambda),
\end{aligned}
$$

where $\tilde{A}=A>0, \tilde{B}=B+2 \theta A>0$ and $\tilde{C}=C+\theta B+\theta^{2} A \geq 0$ for a large enough shift $\theta$. By Theorem 2. condition (2) holds for $(A, B, C)$ if and only if it holds for $(\tilde{A}, \tilde{B}, \tilde{C})($ for any $\theta)$.

The following result about the relationship between hyperbolic QEPs and overdamped QEPs will turn out to be instructive later on.

Theorem 5. A hyperbolic QEP is overdamped if and only if $\lambda_{1} \leq 0$.

Proof. The "only if" part is already known. For the "if" part, we know from the proof of Theorem 3 that $Q(\lambda)=\lambda^{2} A+\lambda B+C \geq 0$ for all $\lambda \geq \lambda_{1}$. In particular, $Q(0)=C \geq 0$. Assume that $B$ is not positive definite. Then $B+2 \theta A$ is singular for some $\theta \geq 0$. Let $x$ be a nonzero vector such that $(B+2 \theta A) x=0$. Then

$$
\left(x^{*}(B+2 \theta A) x\right)^{2}>4\left(x^{*} A x\right)\left(x^{*}\left(C+\theta B+\theta^{2} A\right) x\right)
$$

fails. This contradicts the hyperbolicity of the QEP. Thus, $B>0$.

The next example, taken from [8], illustrates the usefulness of Algorithm [1]

Example 1. We consider the QEP (11) with

$$
A=\left[\begin{array}{ll}
1 & 0 \\
0 & 1
\end{array}\right], B=\epsilon\left[\begin{array}{ll}
1 & 0 \\
0 & 6
\end{array}\right], C=\left[\begin{array}{cc}
\frac{1}{2} & 1 \\
1 & 7
\end{array}\right],
$$

where $\epsilon>0$ is a parameter.

For $\epsilon=0.99$, the four eigenvalues of the QEP are all real with $\lambda_{2}=-0.7788$ and $\lambda_{3}=-1.1824$. However, $Q(\lambda)>0$ for $\lambda=\left(\lambda_{2}+\lambda_{3}\right) / 2$. Thus the QEP is not overdamped at $\epsilon=0.99$.

For the QEP to be overdamped, we have to increase $\epsilon$. For $\epsilon=1.797789047$, the four eigenvalues are all real with $\lambda_{2}=-1.1516555$ and $\lambda_{3}=-1.1516828$, and $Q(\lambda)<0$ for $\lambda=\left(\lambda_{2}+\lambda_{3}\right) / 2$. Thus the QEP is overdamped. For $\epsilon=$ 1.797789046, the QEP has a pair of complex conjugate eigenvalues and the QEP is not overdamped. 
Thus, the QEP is overdamped for $\epsilon \geq 1.797789047$ and is not overdamped for $0<\epsilon \leq 1.797789046$.

If we use the sufficient condition

$$
\left(\lambda_{\min }(B)\right)^{2}>4 \lambda_{\max }(A) \lambda_{\max }(C),
$$

we can only confirm that the QEP is overdamped for $\epsilon \geq 5.35$. (For this and other examples in this paper, all computations are done in Matlab version 5.3 on a Sun workstation.)

When $A, B>0, C \geq 0$, the condition (2) is called the overdamping condition [6]. Our Algorithm 1 thus provides a relatively easy way to check the overdamping con-

dition. The overdamping condition is a sufficient condition for all eigenvalues of the QEP to be real and nonpositive. In this regard, one might ask the following question. If all $2 n$ eigenvalues of the QEP have been found to be real and nonpositive, does it really matter whether the overdamping condition is satisfied? The answer is definitely yes. Indeed, if the overdamping condition is satisfied for the matrices $A, B, C$, then for any matrices $\hat{A}, \hat{B}, \hat{C}$ with $0<\hat{A} \leq A, \hat{B} \geq B, 0 \leq \hat{C} \leq C$, the overdamping condition is still satisfied, and thus all eigenvalues of the corresponding QEP are known to be real and nonpositive without any computation. On the other hand, if we determine that all eigenvalues of the QEP (1) are real and nonpositive using a cheaper method without verifying the overdamping condition, and then the matrices $A, B, C$ are changed to $\hat{A}, \hat{B}, \hat{C}$, there is no guarantee that the eigenvalues will remain real and nonpositive (see Example 1). We would have to apply that cheaper method to check this again.

Moreover, our Algorithm 1 can be modified so that the overdamping condition can be checked by computing at most two eigenvalues. This will be discussed in Section 4 .

\section{SOLVING HYPERBOLIC QEPS}

The standard approach for finding all $2 n$ eigenpairs of the QEP is to use a proper linearization and solve a $2 n \times 2 n$ generalized eigenvalue problem. A common linearization of the QEP (11) is

$$
\lambda\left[\begin{array}{ll}
I & 0 \\
0 & A
\end{array}\right]-\left[\begin{array}{cc}
0 & I \\
-C & -B
\end{array}\right] .
$$

The linearization has the same eigenvalues as the QEP. Moreover, the partial multiplicities of each eigenvalue are the same as those for the matrix polynomial $Q(\lambda)$. The eigenvectors for $Q(\lambda)$ can be obtained by taking the first $n$ components of the eigenvectors of (5). See [21], for example.

We can use the QZ algorithm to find the eigenpairs of the pencil (5). To save computational work, we can reduce the problem of finding the eigenpairs of the pencil (5) to that of finding the eigenpairs of the matrix

$$
\left[\begin{array}{cc}
0 & I \\
-A^{-1} C & -A^{-1} B
\end{array}\right]
$$

by the QR algorithm. However, there may be a loss of accuracy in doing this if $A$ is ill conditioned. Although $A$ is positive definite for hyperbolic QEPs, it may still be ill conditioned. Indeed, in the original paper [6] by Duffin, the matrix $A$ is only required to be positive semidefinite. 
Another approach is based on the factorization of the matrix polynomial $Q(\lambda)$. Indeed, it is well known that $Q(\lambda)$ admits the factorization

$$
Q(\lambda)=(\lambda A+A X+B)(\lambda I-X)
$$

if and only if $X$ is a solution of the corresponding quadratic matrix equation

$$
Q(X)=A X^{2}+B X+C=0
$$

(see [15, Theorem 3.3] or [7, Corollary 3.6]). A solution of (7) is also called a solvent of $Q(X)$. When (7) has a solution $X$, the $2 n$ eigenvalues of $Q(\lambda)$ can be found by finding the eigenvalues of the matrix $X$ and the matrix pencil $\lambda A+A X+B$. This solvent approach has been explored in [4] and [5], and more recently in [9] and [11.

Suppose that $X^{(1)}$ and $X^{(2)}$ are two solvents of $Q(X)$ and the spectra of these two solvents are disjoint. Then the eigenpairs of $Q(\lambda)$ are obtained by gathering the eigenpairs of the two solvents. It is easy to see that the partial multiplicities of an eigenvalue of either solvent are the same as those of this eigenvalue for $Q(\lambda)$. Thus, qualitatively speaking, computing the eigenpairs of $X^{(1)}$ and $X^{(2)}$ is no more difficult than computing the eigenpairs of (5).

The solvent approach will be more efficient than the linearization approach if the solvents $X^{(1)}$ and $X^{(2)}$ exist and can be found efficiently. This is indeed the case for an overdamped QEP, with $X^{(1)}$ and $X^{(2)}$ being the primary and secondary solvents of $Q(X)$, respectively. Note that any hyperbolic QEP can be changed to an overdamped QEP as long as an upper bound for its largest eigenvalue $\lambda_{1}$ is known (see Theorem 5) and that such a bound can be obtained by applying the results in 12. We then limit our attention to overdamped QEPs.

The two solvents can be found efficiently by applying an algorithm based on cyclic reduction (see [3, for example). The algorithm can be introduced as in 19.

From $A X^{2}+B X+C=0$, we get an infinite block tridiagonal system

$$
\left[\begin{array}{cccc}
B & A & & \\
C & B & A & \\
& C & B & \ddots \\
& & \ddots & \ddots
\end{array}\right]\left[\begin{array}{c}
X \\
X^{2} \\
X^{3} \\
\vdots
\end{array}\right]=\left[\begin{array}{c}
-C \\
0 \\
0 \\
\vdots
\end{array}\right]
$$

By recursively applying block cyclic reduction, i.e., an odd-even permutation of block rows and block columns, followed by one step of Gaussian elimination, we obtain the following sequence of infinite block tridiagonal systems:

$$
\left[\begin{array}{cccc}
X_{i} & -A_{i} & & \\
-C_{i} & B_{i} & -A_{i} & \\
& -C_{i} & B_{i} & \ddots \\
& & \ddots & \ddots
\end{array}\right]\left[\begin{array}{c}
X \\
X^{2^{i}+1} \\
X^{2 \cdot 2^{i}+1} \\
\vdots
\end{array}\right]=\left[\begin{array}{c}
-C \\
0 \\
0 \\
\vdots
\end{array}\right], \quad i \geq 1
$$

where the matrices $X_{i}, A_{i}, B_{i}$, and $C_{i}$ are defined by the algorithm we need: 


\section{Algorithm 2.}

$$
\begin{aligned}
& X_{0}=B, \quad A_{0}=A, \quad B_{0}=B, \quad C_{0}=C, \\
& X_{i+1}=X_{i}-A_{i} B_{i}^{-1} C_{i}, \\
& A_{i+1}=A_{i} B_{i}^{-1} A_{i}, \\
& B_{i+1}=B_{i}-A_{i} B_{i}^{-1} C_{i}-C_{i} B_{i}^{-1} A_{i}, \\
& C_{i+1}=C_{i} B_{i}^{-1} C_{i}, \quad i=0,1, \ldots
\end{aligned}
$$

From (9), we get

$$
X_{i} X-A_{i} X^{2^{i}+1}=-C, \quad i \geq 1 .
$$

If, for example, $\rho(X)<1$ and $\left\{\left\|X_{i}^{-1} A_{i}\right\|\right\}$ is bounded (where $\rho(\cdot)$ is the spectral radius and $\|\cdot\|$ is any matrix norm), then we see from (10) that $-X_{i}^{-1} C$ converges to $X$ rapidly.

The presentation of Algorithm 2 here is slightly different from that in 3 . We would have $A_{i+1}=-A_{i} B_{i}^{-1} A_{i}$ and $C_{i+1}=-C_{i} B_{i}^{-1} C_{i}$ in Algorithm 2 if we follow [3] exactly. However, this difference has no effect on the sequences $\left\{X_{i}\right\}$ and $\left\{B_{i}\right\}$. When the algorithm is well defined, it is easily seen that the matrices $A_{i}, B_{i}$ and $C_{i}$ are all Hermitian.

Most of the analysis of this algorithm can be found in 3. For general matrices $A, B, C$, the algorithm may break down (a singular $B_{i}$ is encountered). Some conditions are given in [3] to guarantee the invertibility of the matrices $B_{i}$. However, it is not easy to verify those conditions for overdamped QEPs. We will now show that the algorithm is well defined for all overdamped QEPs.

It is well known that the QEP (with $A, B>0, C \geq 0$ ) is overdamped if and only if $B>k A+k^{-1} C$ for some real $k>0$. (This follows readily from Theorem 2, It is natural to examine whether the overdamping condition is kept during the iteration.

Lemma 6. Algorithm 2 will not break down for any overdamped QEP. Moreover,

$$
A_{i}>0, C_{i} \geq 0, B_{i}>k^{2^{i}} A_{i}+k^{-2^{i}} C_{i}
$$

for all $i \geq 0$.

Proof. The proof is by induction. We know that (11) is true for $i=0$. We assume (11) is true for $i(i \geq 0)$. So, $B_{i}>0$ and thus $A_{i+1}, B_{i+1}, C_{i+1}$ are defined and $A_{i+1}>0, C_{i+1} \geq 0$. Now,

$$
\begin{aligned}
B_{i+1}= & B_{i}-A_{i} B_{i}^{-1} C_{i}-C_{i} B_{i}^{-1} A_{i} \\
= & B_{i}-\left(k^{2^{i}} A_{i}+k^{-2^{i}} C_{i}\right) B_{i}^{-1}\left(k^{2^{i}} A_{i}+k^{-2^{i}} C_{i}\right) \\
& +k^{2^{i+1}} A_{i} B_{i}^{-1} A_{i}+k^{-2^{i+1}} C_{i} B_{i}^{-1} C_{i} \\
> & k^{2^{i+1}} A_{i+1}+k^{-2^{i+1}} C_{i+1},
\end{aligned}
$$

where we have used the fact that $X-Y X^{-1} Y>Y-Y Y^{-1} Y=0$ whenever $X>Y>0$.

This lemma implies that the QEPs $Q_{i}(\lambda)(x)=\left(\lambda^{2} A_{i}+\lambda B_{i}+C_{i}\right) x=0$ are all overdamped. 
Theorem 7. Let the $Q E P$ (11) be overdamped, $X^{(1)}$ and $X^{(2)}$ be the primary and secondary solvents of $Q(X)$, respectively. Then the sequence $\left\{X_{i}\right\}$ in Algorithm 2 converges quadratically to a nonsingular matrix $\hat{X}$ with

$$
\limsup _{i \rightarrow \infty} \sqrt[2^{i}]{\left\|X_{i}-\hat{X}\right\|}=\lambda_{n} / \lambda_{n+1}<1
$$

where $\|\cdot\|$ is any matrix norm. Moreover,

$$
X^{(1)}=-\hat{X}^{-1} C, \quad X^{(2)}=-A^{-1} \hat{X}^{*} .
$$

Proof. We apply the results on cyclic reduction as presented in [3] Let $\theta=\left(\lambda_{n}+\right.$ $\left.\lambda_{n+1}\right) / 2$. We have

$$
\theta A\left(\theta^{-1} X^{(1)}\right)^{2}+B\left(\theta^{-1} X^{(1)}\right)+\theta^{-1} C=0
$$

and

$$
\theta A+B\left(\theta\left(X^{(2)}\right)^{-1}\right)+\theta^{-1} C\left(\theta\left(X^{(2)}\right)^{-1}\right)^{2}=0 .
$$

Note that $\rho\left(\theta^{-1} X^{(1)}\right)<1$ and $\rho\left(\theta\left(X^{(2)}\right)^{-1}\right)<1$. We now apply Algorithm 2 with the triple $(A, B, C)$ replaced by $\left(\theta A, B, \theta^{-1} C\right)$ (which also satisfies the overdamping condition) and generate the sequences $\left\{\tilde{X}_{i}\right\},\left\{\tilde{A}_{i}\right\},\left\{\tilde{B}_{i}\right\}$, and $\left\{\tilde{C}_{i}\right\}$. It is readily seen that for all $i \geq 0$,

$$
\tilde{X}_{i}=X_{i}, \tilde{A}_{i}=\theta^{2^{i}} A_{i}, \tilde{B}_{i}=B_{i}, \tilde{C}_{i}=\theta^{-2^{i}} C_{i} .
$$

By Theorem 16 of [3], the sequences $\left\{\left\|B_{i}\right\|\right\},\left\{\left\|B_{i}^{-1}\right\|\right\}$ are bounded and

$$
\limsup _{i \rightarrow \infty} \sqrt[2^{i}]{\left\|\tilde{A}_{i}\right\|} \leq \rho\left(\theta\left(X^{(2)}\right)^{-1}\right), \limsup _{i \rightarrow \infty} \sqrt[2^{i}]{\left\|\tilde{C}_{i}\right\|} \leq \rho\left(\theta^{-1} X^{(1)}\right) .
$$

In view of (10), we have for each $i \geq 1$,

$$
\theta^{-1} C+X_{i}\left(\theta^{-1} X^{(1)}\right)-\tilde{A}_{i}\left(\theta^{-1} X^{(1)}\right)^{2^{i}+1}=0 .
$$

We now define a new sequence $\left\{Y_{i}\right\}$ by

$$
Y_{0}=B, \quad Y_{i+1}=Y_{i}-C_{i} B_{i}^{-1} A_{i}, \quad i=0,1, \ldots
$$

Then on the one hand, we have $Y_{i}=X_{i}^{*}$; on the other hand, we get the analog of (15):

$$
\theta A+Y_{i}\left(\theta\left(X^{(2)}\right)^{-1}\right)-\tilde{C}_{i}\left(\theta\left(X^{(2)}\right)^{-1}\right)^{2^{i}+1}=0 .
$$

Since $\lim \tilde{C}_{i}\left(\theta\left(X^{(2)}\right)^{-1}\right)^{2^{i}+1}=0$ and $A$ is nonsingular, it follows from (16) that $\left\{Y_{i}\right\}$ converges to the nonsingular matrix $\hat{Y}=-A X^{(2)}$. Moreover,

$$
\begin{aligned}
\limsup _{i \rightarrow \infty} \sqrt[2^{i}]{\left\|Y_{i}-\hat{Y}\right\|} & =\limsup _{i \rightarrow \infty} \sqrt[2^{i}]{\left\|\theta^{-1} \tilde{C}_{i}\left(\theta\left(X^{(2)}\right)^{-1}\right)^{2^{i}+1} X^{(2)}\right\|} \\
& \leq \limsup _{i \rightarrow \infty} \sqrt[2^{i}]{\left\|\tilde{C}_{i}\right\|} \limsup _{i \rightarrow \infty} \sqrt[2^{i}]{\left\|\left(\theta\left(X^{(2)}\right)^{-1}\right)^{2^{i}}\right\|} \\
& \leq \rho\left(\theta^{-1} X^{(1)}\right) \rho\left(\theta\left(X^{(2)}\right)^{-1}\right)=\lambda_{n} / \lambda_{n+1}<1 .
\end{aligned}
$$

Therefore, $\left\{X_{i}\right\}$ converges to the nonsingular matrix $\hat{X}=\hat{Y}^{*}$ and (12) holds. Since $\hat{X}^{*}=\hat{Y}=-A X^{(2)}$, we get $X^{(2)}=-A^{-1} \hat{X}^{*}$. Letting $i \rightarrow \infty$ in (15), we get $X^{(1)}=-\hat{X}^{-1} C$. 
Corollary 8. The primary solution $X^{(1)}$ and the secondary solution $X^{(2)}$ are related by

$$
X^{(1)}=A^{-1}\left(\left(X^{(2)}\right)^{*}\right)^{-1} C .
$$

Proof. The relation is obtained by eliminating $\hat{X}$ in (13).

Before we apply Algorithm 2 to approximate the matrix $\hat{X}$, we have to specify how the matrix products in the algorithm are computed and what the stopping criterion is. We assume that the matrices $A, B, C$ are $n \times n$ real symmetric matrices. For each iteration, we need to compute $A_{i} B_{i}^{-1} C_{i}$ (note that $C_{i} B_{i}^{-1} A_{i}=\left(A_{i} B_{i}^{-1} C_{i}\right)^{T}$ ) and the symmetric matrices $A_{i} B_{i}^{-1} A_{i}$ and $C_{i} B_{i}^{-1} C_{i}$. These matrices can be computed as follows. Let $B_{i}=L_{i} L_{i}^{T}$ be the Cholesky factorization of $B_{i}, V_{i}=L_{i}^{-1} A_{i}$, and $W_{i}=L_{i}^{-1} C_{i}$. Then $A_{i} B_{i}^{-1} C_{i}=V_{i}^{T} W_{i}, A_{i} B_{i}^{-1} A_{i}=V_{i}^{T} V_{i}$, and $C_{i} B_{i}^{-1} C_{i}=$ $W_{i}^{T} W_{i}$. Therefore, the computational work required for one iteration of Algorithm2 2 is about $\frac{19}{3} n^{3}$ flops. The algorithm is stopped when $\left\|X_{i+1}-X_{i}\right\|_{\infty} /\left\|X_{i+1}\right\|_{\infty}<\eta$, a prescribed tolerance, and $X_{i+1}$ is taken to be an approximation to $\hat{X}$. Unless $\lambda_{n} / \lambda_{n+1}$ is very close to 1 , usually 10 iterations of Algorithm 2 is enough to approximate $\hat{X}$ with high precision, thanks to the quadratic convergence given by (12).

In a practical implementation of Algorithm 2, we should also guard against the possibility of overflow during the computation. In the proof of Theorem 7, we have already seen that if the triple $(A, B, C)$ in Algorithm 2 is replaced by $\left(\theta A, B, \theta^{-1} C\right)$, then the relations (14) hold and the sequences $\left\{\tilde{A}_{i}\right\}$ and $\left\{\tilde{C}_{i}\right\}$ converge to zero. In practice, the value of $\theta$ is not known beforehand and Algorithm 2 is applied to the triple $(A, B, C)$, rather than to $\left(\theta A, B, \theta^{-1} C\right)$. In this case, it is possible that one of the sequences $\left\{A_{i}\right\}$ and $\left\{C_{i}\right\}$ grows unbounded although $\left\|A_{i}\right\|\left\|C_{i}\right\|=\left\|\tilde{A}_{i}\right\|\left\|\tilde{C}_{i}\right\|$ converges to zero. This problem is easily solved by adding a balancing procedure at the end of each iteration. More precisely, at the end of the $i$ th iteration (in which $A_{i}$ and $C_{i}$ are obtained), we do the following:

$$
\alpha_{i}=\sqrt{\left\|C_{i}\right\|_{\infty} /\left\|A_{i}\right\|_{\infty}}, \quad A_{i} \leftarrow \alpha_{i} A_{i}, \quad C_{i} \leftarrow C_{i} / \alpha_{i} .
$$

The balancing procedure ensures that $\left\|A_{i}\right\|_{\infty}=\left\|C_{i}\right\|_{\infty}$, and thus both sequences $\left\{A_{i}\right\}$ and $\left\{C_{i}\right\}$ converge to zero. Note that the balancing procedure does not change the sequences $\left\{X_{i}\right\}$ and $\left\{B_{i}\right\}$ and that the cost of balancing is only $O\left(n^{2}\right)$.

In terms of computational work, the comparison between the solvent approach and the linearization approach depends on several factors: (a) Do we use QR or QZ? (b) Do we need eigenvectors along with eigenvalues? (c) How many iterations are needed for Algorithm 22? If the QZ algorithm is needed for the linearization approach and/or both eigenvalues and eigenvectors are needed, then the computational work for the solvent approach is typically less than $50 \%$ that of the linearization approach. If the QR algorithm is used for both approaches and only eigenvalues are needed, then the solvent approach is less expensive when Algorithm 2 requires at most 12 iterations. It will be seen in the next section that the solvent approach can also be used to check the overdamping condition more efficiently for any QEP with $A, B>0$ and $C \geq 0$.

For overdamped QEPs, there are situations in which the QZ algorithm is needed for the linearization approach, while the QR algorithm is adequate for the solvent approach. Indeed, the matrix $\hat{X}$ is often well conditioned when $B$ is well conditioned, even if $A$ and $C$ are both ill conditioned. Thus, we may apply the 
QR algorithm to the matrices $-\hat{X}^{-1} C$ and $-\left(\hat{X}^{*}\right)^{-1} A$ to get the eigenpairs of $X^{(1)}=-\hat{X}^{-1} C$ and $X^{(2)}=\left(-\left(\hat{X}^{*}\right)^{-1} A\right)^{-1}$ (see Theorem 7). Of course, we can still apply the QZ algorithm to the pencils $\lambda \hat{X}+C$ and $\lambda A+\hat{X}^{*}$ to get possibly higher accuracy.

Example 2. We consider the QEP (11) with $n=8$ and

$$
A=(1 /(i+j-1)), \quad B=\operatorname{tridiag}(-1,6,-1), \quad C=(1 /(i+j)) .
$$

The QEP is overdamped with $\operatorname{cond}_{2}(A)=1.5 \times 10^{10}$, $\operatorname{cond}_{2}(C)=5.6 \times 10^{10}$, and $\operatorname{cond}_{2}(B)=1.9$. We compute the 16 eigenvalues by the QZ algorithm for the linearization approach, and by the QR algorithm for the solvent approach. For the solvent approach, the matrix $\hat{X}$ is computed by Algorithm 2 in 5 iterations (with $\eta=10^{-12}$ for the stopping criterion), and we have $\operatorname{cond}_{2}(\hat{X})=2.1$. The eigenvalues from both methods match very well. For example, for both methods we have

$$
\lambda_{8}=-0.31548025829685, \quad \lambda_{9}=-2.35473981343162 .
$$

If we use the QR algorithm in the linearization approach, we get

$$
\lambda_{8}=-0.31548040167845, \quad \lambda_{9}=-2.35473975092443 .
$$

Note that the last 8 digits are changed for each eigenvalue.

Algorithm 2 provides an approximation to the matrix $\hat{X}$. The accuracy of this approximation is dependent on the conditioning of the matrices $B_{i}$ in the algorithm. Although Lemma 6] shows that all these matrices are positive definite, they can still be ill conditioned. Indeed, from the proof of Theorem 16 of [3] and the proof of Theorem 7 we can see that the condition numbers of these matrices have an upper bound that is dependent on the ratio $\lambda_{n} / \lambda_{n+1}$. If this ratio approaches 1 , the upper bound goes to infinity. This means that the matrices $B_{i}$ are probably ill conditioned for large $i$ when $\lambda_{n} / \lambda_{n+1}$ is very close to 1 , even if $B_{0}$ is well conditioned. However, the accuracy of the eigenvalues computed by the solvent approach is typically no worse than that achieved by the linearization approach. The reason for this is that in the limit case $\lambda_{n}=\lambda_{n+1}, \lambda_{n}$ is typically a double eigenvalue with partial multiplicity 2 . Therefore, when $\lambda_{n} / \lambda_{n+1}$ is very close to 1 , we can only expect half of the machine precision for the linearization approach. For the solvent approach, the eigenvalues $\lambda_{n}$ and $\lambda_{n+1}$ are obtained separately from $X^{(1)}$ and $X^{(2)}$, although the accuracy will be affected by the accuracy of $\hat{X}$.

Example 3. We consider the QEP (11) with $n=2$ and

$$
A=W I_{2} W^{T}, \quad B=W\left((4+\epsilon) I_{2}\right) W^{T}, \quad C=W\left[\begin{array}{ll}
2 & 2 \\
2 & 2
\end{array}\right] W^{T},
$$

where $W=\left[\begin{array}{ll}1 & 2 \\ 3 & 4\end{array}\right]$ is introduced to make the data less simplified. The QEP is overdamped for $\epsilon>0$, and the exact eigenvalues are

$$
0, \frac{-(4+\epsilon) \pm \sqrt{8 \epsilon+\epsilon^{2}}}{2},-(4+\epsilon) \text {. }
$$

We first compute the 4 eigenvalues by the solvent approach. The matrix $\hat{X}$ is computed by Algorithm 2 in 25 iterations (with $\eta=10^{-12}$ for the stopping criterion), 
and we have $\operatorname{cond}_{2}\left(B_{24}\right)=3.5 \times 10^{5}$. The eigenvalues are then found from $X^{(1)}$ and $X^{(2)}$ by the QR algorithm. They are

$$
\begin{array}{ll}
-3.10863 \times 10^{-15}, & -1.9999985860894, \\
-2.0000014139116, & -4.0000000000010 .
\end{array}
$$

For the linearization approach, the 4 eigenvalues are obtained by applying the QR algorithm to (6). They are

$$
\begin{array}{cc}
3.33067 \times 10^{-15}, & -1.9999985876094, \\
-2.0000014123916, & -4.0000000000010 .
\end{array}
$$

We have also used the QZ algorithm for the linearization approach, but the results are not any better. This suggests that the accuracy of the linearization approach is affected by the closeness of $\lambda_{2}$ and $\lambda_{3}$, rather than by the conditioning of $A$. For this example, we have $\lambda_{2} / \lambda_{3}=0.9999986$ and $\operatorname{cond}_{2}(A)=223$. Since the exact values for $\lambda_{2}$ and $\lambda_{3}$ are $-1.99999858578 \cdots,-2.00000141421 \cdots$, respectively, the solvent approach provides better accuracy for these two eigenvalues.

We end this section by a comment on the convergence rate of Algorithm 2, By Theorem 7 , the convergence rate is determined by the ratio $\lambda_{n} / \lambda_{n+1}$. Theorem $[5$ is now instructive. By that theorem, new QEPs obtained by a shift in the eigenvalue parameter are still overdamped as long as we still have $\lambda_{1} \leq 0$ after the shift. The computational work involved in changing one QEP into another by a shift is only $O\left(n^{2}\right)$. We will have faster convergence for Algorithm 2 if we apply it to a new QEP obtained by shifting the eigenvalues towards the origin. Thus, for a given overdamped QEP, the best possible ratio is $\left(\lambda_{n}-\lambda_{1}\right) /\left(\lambda_{n+1}-\lambda_{1}\right)$ when the shift technique is used. Although the exact value of $\lambda_{1}$ is not known in practice, a good upper bound for $\lambda_{1}$ works just fine. Such an upper bound can sometimes be found by using the results in [12. Better upper bounds may be obtained inexpensively by approximating $\lambda_{1}$ using a linearization and the Arnoldi method (see [20]) when, for example, $A=I$ and $B, C$ are sparse. (Algorithm 2 does not exploit any sparse structure for the matrices $A, B$, and $C$, and requires more than $6 n^{3}$ flops each iteration.) If a negative number $b$ is an upper bound for $\lambda_{1}$, we can shift the eigenvalues to the right by $|b|(\lambda \leftarrow \lambda+|b|)$ to improve convergence. Shifting the eigenvalues to the right (left) by a large step can speed up (slow down) the convergence of Algorithm 2 significantly.

Example 4. For Example 1 with $\epsilon=2, \hat{X}$ can be obtained by Algorithm 2 in 7 iterations with $\eta=10^{-12}$. If we obtain a new QEP by shifting the eigenvalues to the left by $100(\lambda \leftarrow \lambda-100)$ and apply Algorithm 2 to the new QEP, then 13 iterations will be needed.

\section{Testing FOR HYPERBolicity By COMPUting AT MOst TWO Eigenvalues}

Testing for hyperbolicity is the same as testing for the overdamping condition after a proper shift has been applied to the eigenvalue parameter. So, once again, we limit our attention to the QEP (1) with $A, B>0$ and $C \geq 0$. Algorithm 2 and Theorem 7 provide a method for checking the overdamping condition by computing at most two eigenvalues.

The idea is very simple. We apply Algorithm 2 to the QEP and use the Cholesky factorization of $B_{i}$ in the computation. If $B_{i}$ does not have a Cholesky factorization for some $i$, then $B_{i}$ is not positive definite and thus the QEP is not overdamped. 
If all matrices $B_{i}$ have Cholesky factorization during the iteration in Algorithm 2 but the sequence $\left\{X_{i}\right\}$ does not converge (quickly) to a nonsingular matrix $\hat{X}$, then the QEP is not overdamped. We now assume that $\left\{X_{i}\right\}$ converges (quickly) to a nonsingular matrix $\hat{X}$. Then we compute the eigenvalue $\lambda^{(1)}$ of $-\hat{X}^{-1} C$ with the largest modulus $\left(\lambda^{(1)}\right.$ is the candidate for $\lambda_{n}$ ). If $\lambda^{(1)}$ is not a nonpositive real number, then the QEP is not overdamped. If $\lambda^{(1)}$ is a nonpositive real number, then we compute the eigenvalue $1 / \lambda^{(2)}$ of $-\left(\hat{X}^{*}\right)^{-1} A$ with the largest modulus $\left(\lambda^{(2)}\right.$ is the candidate for $\lambda_{n+1}$ ). If $\lambda^{(2)}$ is not a negative real number, then the QEP is not overdamped. If $\lambda^{(2)}$ is a negative real number and $\lambda^{(1)}>\lambda^{(2)}$ (otherwise the QEP is not overdamped), then the QEP is overdamped if and only if $Q\left(\left(\lambda^{(1)}+\lambda^{(2)}\right) / 2\right)<0$.

We mention that it is possible to obtain good approximations for $\lambda^{(1)}$ and $\lambda^{(2)}$ without computing all the eigenvalues of the two matrices. Significant saving may be achieved by using the Arnoldi method to approximate these two eigenvalues, when $n$ is relatively large (say $n=1000$ ). See [20], for example. We also note that the computed values $\lambda^{(1)}$ and $\lambda^{(2)}$ need not be very accurate for the ultimate test $Q\left(\left(\lambda^{(1)}+\lambda^{(2)}\right) / 2\right)<0$. What we need is $\left(\lambda^{(1)}+\lambda^{(2)}\right) / 2 \in\left(\lambda_{n+1}, \lambda_{n}\right)$ when the QEP is actually overdamped.

The above procedure is particularly useful in confirming that a given QEP is not overdamped. In fact, when the QEP is not overdamped, $B_{i}>0$ typically fails for a small $i$, so no eigenvalue computation is necessary. We do not know if there are any examples of nonoverdamped QEPs for which $B_{i}>0$ for all $i$. It may be safe to say that $B_{i}>0$ for all $i \leq 20$ is a strong indication that the QEP is overdamped or nearly overdamped. Of course, to be certain, we have to follow the procedure to the end in order to confirm that a given QEP is overdamped.

Example 5. We consider the QEP (11), where $A=I$,

$$
B=\epsilon\left[\begin{array}{cccccc}
20 & -10 & & & & \\
-10 & 30 & -10 & & & \\
& -10 & 30 & -10 & & \\
& & -10 & \ddots & \ddots & \\
& & & \ddots & 30 & -10 \\
& & & & -10 & 20
\end{array}\right] \text {, }
$$

and

$$
C=\left[\begin{array}{ccccc}
15 & -5 & & & \\
-5 & 15 & -5 & & \\
& -5 & \ddots & \ddots & \\
& & \ddots & \ddots & -5 \\
& & & -5 & 15
\end{array}\right]
$$

are $100 \times 100$ matrices, and $\epsilon>0$ is a parameter. This is the example used in [11] when $\epsilon=1$.

For $\epsilon=0.5196152423$, the sequence $\left\{X_{i}\right\}$ from Algorithm 2 converges in 21 iterations $\left(\left\|X_{21}-X_{20}\right\|_{\infty} /\left\|X_{21}\right\|_{\infty}<\eta=10^{-12}\right)$. We then take $\hat{X}=X_{21}$ and find that $\lambda^{(1)}=-2.886723, \lambda^{(2)}=-2.886779$, and $Q(\lambda)<0$ for $\lambda=\left(\lambda^{(1)}+\right.$ $\left.\lambda^{(2)}\right) / 2$. Thus the QEP is overdamped. For $\epsilon=0.5196152422, B_{17}>0$ fails for Algorithm 2, so the QEP is not overdamped. Therefore, the QEP is overdamped for $\epsilon \geq 0.5196152423$ and is not overdamped for $0<\epsilon \leq 0.5196152422$. The exact 
turning point is some $\epsilon_{*}$ in the interval [0.5196152422, 0.5196152423]. Normally, the convergence of Algorithm 2 is faster as $\epsilon$ moves away from $\epsilon_{*}$ to the right. For example, for $\epsilon=0.519616,0.5197,0.52,1,\left\{X_{i}\right\}$ converges in 14, 11, 10, 5 iterations, respectively. Also, it is easier to confirm that the QEP is not overdamped as $\epsilon$ moves away from $\epsilon_{*}$ to the left. For example, for $\epsilon=0.51961524,0.519615,0.5196,0.51$, we do not have $B_{15}>0, B_{11}>0, B_{8}>0, B_{4}>0$, respectively. By the way, $B_{1}>0$ fails for Example 1 with $\epsilon=0.99$.

\section{A NEW MEASURE FOR THE DEGREE OF HYPERBOLICITY}

Once a QEP has been determined to be hyperbolic, the distance of this QEP to the nearest nonhyperbolic QEP can be defined (see [10] and 13]). However, the computation of this distance is very expensive. Without computing this distance, we can still define the degree of hyperbolicity. As noted in [13, for a hyperbolic QEP, a natural measure of the degree of hyperbolicity is the quantity

$$
\min _{\|x\|_{2}=1} \sqrt{\left(x^{*} B x\right)^{2}-4\left(x^{*} A x\right)\left(x^{*} C x\right)} \text {. }
$$

However, this quantity is still very difficult to compute. Here we introduce a new measure for the degree of hyperbolicity. The measure is based on the following result.

Theorem 9. Consider the $Q E P$ (1) with $A, B, C \in \mathcal{H}_{n}$ and $A>0$. Let $f(x)=$ $\left(x^{*} B x\right)^{2}-4\left(x^{*} A x\right)\left(x^{*} C x\right)$. If

$$
\min _{\|x\|_{2}=1} f(x)=f\left(x_{0}\right)=0
$$

then the QEP has $2 n$ real eigenvalues with $\lambda_{1} \geq \cdots \geq \lambda_{n}=\lambda_{n+1} \geq \cdots \geq \lambda_{2 n}$. Moreover,

and $x_{0}$ is a corresponding eigenvector.

$$
\lambda_{n}=\lambda_{n+1}=-\frac{x_{0}^{*} B x_{0}}{2 x_{0}^{*} A x_{0}}
$$

Proof. Without loss of generality, we assume that $B>0$ and $C \geq 0$. For any $\epsilon>0$, $\hat{Q}(\lambda)=\lambda^{2} A+\lambda(B+\epsilon I)+C$ is overdamped. Its $2 n$ eigenvalues are

$$
\lambda_{1}(\epsilon) \geq \cdots \geq \lambda_{n}(\epsilon)>\lambda_{n+1}(\epsilon) \geq \cdots \geq \lambda_{2 n}(\epsilon) .
$$

By Theorem 3 .

$$
Q\left(\left(\lambda_{n}(\epsilon)+\lambda_{n+1}(\epsilon)\right) / 2\right)<0 .
$$

By the proof of Theorem 7.7 in [15,

$$
\frac{-b-\sqrt{b^{2}-4 a c}}{2 a} \leq \lambda_{n+1}(\epsilon)<\lambda_{n}(\epsilon) \leq \frac{-b+\sqrt{b^{2}-4 a c}}{2 a},
$$

where

$$
a=x_{0}^{*} A x_{0}, \quad b=x_{0}^{*}(B+\epsilon I) x_{0}, \quad c=x_{0}^{*} C x_{0} .
$$

Letting $\epsilon \rightarrow 0$ in (17)-(19), we conclude that $\lambda_{1} \geq \cdots \geq \lambda_{n}=\lambda_{n+1} \geq \cdots \geq \lambda_{2 n}$, $Q\left(\lambda_{n}\right) \leq 0$, and

$$
\lambda_{n}=\lambda_{n+1}=-\frac{x_{0}^{*} B x_{0}}{2 x_{0}^{*} A x_{0}} .
$$

Since $x_{0}^{*}\left(-Q\left(\lambda_{n}\right)\right) x_{0}=0$ and $-Q\left(\lambda_{n}\right) \geq 0$, we have $Q\left(\lambda_{n}\right) x_{0}=0$, i.e., $x_{0}$ is an eigenvector corresponding to $\lambda_{n}$. 
Theorem 9 suggests that the gap between the primary and the secondary eigenvalues can be used as a measure for the degree of hyperbolicity. We propose the new measure as follows: If the QEP is hyperbolic, then the number

$$
d=\frac{1}{4}\left(\lambda_{n+1}-\lambda_{n}\right)^{2}
$$

is a measure of the degree of hyperbolicity. If $d$ is large, we may say that the QEP is highly hyperbolic; if $d$ is close to 0, we may say that the QEP is marginally hyperbolic. Note that the number $d$ is obtained immediately once a QEP has been confirmed to be hyperbolic by computing the eigenvalues $\lambda_{n}$ and $\lambda_{n+1}$.

To define the distance of a hyperbolic QEP to the nearest nonhyperbolic QEP, we ask by how much the coefficient matrices must be perturbed for the hyperbolicity to be lost. In 10] and 13, the perturbations are measured in the absolute sense. The degree of hyperbolicity we have defined is invariant if we change $Q(\lambda)$ to $Q(\lambda+c)$. More importantly, it is invariant if we change $Q(\lambda)$ to $c Q(\lambda)(c \neq 0)$. Therefore, to make a connection between our new measure and the distance studied in [10] and [13, the perturbations of coefficient matrices will have to be measured in the relative sense.

When $d=0$, the QEP is "weakly hyperbolic" in the terminology of Markus [18. By Theorem 31.10 of [18, each elementary divisor of the multiple eigenvalue $\lambda_{n}$ has degree at most two. The case for which the multiple eigenvalue $\lambda_{n}$ has at least one elementary divisor of degree two is generic, although there are simple examples for which $\lambda_{n}$ is still semisimple.

The new measure puts the emphasis on hyperbolic QEPs that are nearly nonhyperbolic. Suppose we have a weakly hyperbolic QEP with $\lambda_{n}=\lambda_{n+1}$. We can perturb the triple $(A, B, C)$ to $(\tilde{A}, \tilde{B}, \tilde{C})$ so that the corresponding QEP is hyperbolic. We assume that the multiple eigenvalue $\lambda_{n}$ has at least one elementary divisor of degree two and that neither of $B$ and $C$ is the zero matrix. Let $\Delta A=$ $\tilde{A}-A, \Delta B=\tilde{B}-B, \Delta C=\tilde{C}-C$, and let $\|\cdot\|_{F}$ be the Frobenius norm. So if

$$
\epsilon=\left\|\left(\frac{\Delta A}{\|A\|_{F}}, \frac{\Delta B}{\|B\|_{F}}, \frac{\Delta C}{\|C\|_{F}}\right)\right\|_{F},
$$

we expect to have $\tilde{\lambda}_{n} \sim \lambda_{n}+c_{1} \epsilon^{1 / 2}$ and $\tilde{\lambda}_{n+1} \sim \lambda_{n+1}-c_{2} \epsilon^{1 / 2}$ for the new QEP when $\epsilon$ is small. We simplify the situation by assuming that $c_{1}=c_{2}=1$. It would be reasonable to say that the degree of hyperbolicity of the QEP given by $(\tilde{A}, \tilde{B}, \tilde{C})$ is $\epsilon$. To recover this $\epsilon$ from $\tilde{\lambda}_{n}$ and $\tilde{\lambda}_{n+1}$, we can simply compute $\frac{1}{4}\left(\tilde{\lambda}_{n+1}-\tilde{\lambda}_{n}\right)^{2}$. Although the factor $\frac{1}{4}$ in (20) is somewhat artificial, the square sign is also suggested by Examples 11, 3 and 5. For Example 1, the QEP is not overdamped for $\epsilon=1.797789046$; it is overdamped for $\epsilon=1.797789047$ with $\lambda_{n}=$ -1.1516555 and $\lambda_{n+1}=-1.1516828$. For Example 5 the QEP is not overdamped for $\epsilon=0.5196152422$; it is overdamped for $\epsilon=0.5196152423$ with $\lambda_{n}=-2.886723$ and $\lambda_{n+1}=-2.886779$. For Example 3, the QEP is hyperbolic for all $\epsilon>0$ and the hyperbolicity is lost when $\epsilon=0$. It is easily seen that $d=\frac{1}{4}\left(\lambda_{n+1}-\lambda_{n}\right)^{2} \sim$ $2 \epsilon(\epsilon \rightarrow 0)$ for this example.

\section{REFERENCES}

1. L. Barkwell and P. Lancaster, Overdamped and gyroscopic vibrating systems, Trans. ASME J. Appl. Mech. 59 (1992), 176-181. MR.1176288 (93f:70021) 
2. L. Barkwell, P. Lancaster, and A. S. Markus, Gyroscopically stabilized systems: a class of quadratic eigenvalue problems with real spectrum, Canad. J. Math. 44 (1992), 42-53. MR.1152665 (93a:47015)

3. D. A. Bini, L. Gemignani, and B. Meini, Computations with infinite Toeplitz matrices and polynomials, Linear Algebra Appl. 343-344 (2002), 21-61. MR.1878936 (2002m:65038)

4. G. J. Davis, Numerical solution of a quadratic matrix equation, SIAM J. Sci. Stat. Comput. 2 (1981), 164-175. MR0622713 (82f:65036)

5. J. E. Dennis, Jr., J. F. Traub, and R. P. Weber, Algorithms for solvents of matrix polynomials, SIAM J. Numer. Anal. 15 (1978), 523-533. MR0471278 (57:11015)

6. R. J. Duffin, A minimax theory for overdamped networks, J. Rational Mech. Anal. 4 (1955), 221-233. MR0069030 (16:979b)

7. I. Gohberg, P. Lancaster, and L. Rodman, Matrix polynomials, Academic Press, New York, 1982. MR:0662418 (84c:15012)

8. I. Gohberg, P. Lancaster, and L. Rodman, Quadratic matrix polynomials with a parameter, Adv. Appl. Math. 7 (1986), 253-281. MR0858906 (88e:47027)

9. C.-H. Guo, Numerical solution of a quadratic eigenvalue problem, Linear Algebra Appl. 385 (2004), 391-406. MR2063362

10. Y. Hachez and P. Van Dooren, Elliptic and hyperbolic quadratic eigenvalue problems and associated distance problems, Linear Algebra Appl. 371 (2003), 31-44. MR1997361 (2004g:15011)

11. N. J. Higham and H.-M. Kim, Numerical analysis of a quadratic matrix equation, IMA J. Numer. Anal. 20 (2000), 499-519. MR1795295 (2001i:65054)

12. N. J. Higham and F. Tisseur, Bounds for eigenvalues of matrix polynomials, Linear Algebra Appl. 358 (2003), 5-22. MR1942721 (2003k:15020)

13. N. J. Higham, F. Tisseur, and P. M. Van Dooren, Detecting a definite Hermitian pair and a hyperbolic or elliptic quadratic eigenvalue problem, and associated nearness problems, Linear Algebra Appl. 351-352 (2002), 455-474. MR1917487 (2003f:93047)

14. I. Krupnik, A. Markus, and P. Lancaster, Factorization of selfadjoint quadratic matrix polynomials with real spectrum, Linear Multilinear Algebra 39 (1995), 263-272. MR1365446 (97b:15015)

15. P. Lancaster, Lambda-matrices and vibrating systems, Pergamon Press, Oxford, 1966, and 2nd Edition, Dover, 2002. MR.1949393 (2003i:34016)

16. P. Lancaster, Quadratic eigenvalue problems, Linear Algebra Appl. 150 (1991), 499-506.

17. P. Lancaster, A. S. Markus, and F. Zhou, A wider class of stable gyroscopic systems, Linear Algebra Appl. 370 (2003), 257-267. MR1994333 (2004g:70039)

18. A. S. Markus, Introduction to the spectral theory of polynomial operator pencils, AMS, Providence, RI, 1988. MR0971506 (89h:47023)

19. B. Meini, Efficient computation of the extreme solutions of $X+A^{*} X^{-1} A=Q$ and $X-$ $A^{*} X^{-1} A=Q$, Math. Comp. 71 (2002), 1189-1204. MR1898750 (2003c:15018)

20. D. C. Sorensen, Implicit application of polynomial filters in a k-step Arnoldi method, SIAM J. Matrix Anal. Appl. 13 (1992), 357-385. MR.1146670 (92i:65076)

21. F. Tisseur and K. Meerbergen, The quadratic eigenvalue problem, SIAM Rev. 43 (2001), 235-286. MR1861082 (2002i:65042)

Department of Mathematics and Statistics, University of Regina, Regina, SaskatchEWAN, CANADA S4S 0A2

E-mail address: chguo@math.uregina.ca

Department of Mathematics and Statistics, University of Calgary, Calgary, AlBerta, CANADA T2N $1 \mathrm{~N} 4$

E-mail address: lancaste@ucalgary.ca 